\title{
Mathematical Analysis of Frequency Modulation Symmetric Polarized Diversity Phase-locked Loop
}

\author{
Zhenzhou $\mathrm{Li}^{1, \text { a }}$, Yongming Nie $\mathrm{i}^{1, \mathrm{~b}^{*}}$ and Lujun Zhou ${ }^{1, \mathrm{c}}$ \\ ${ }^{1}$ China Satellite Maritime Tracking and Control Department, Jiangyin, Jiangsu, China \\ ayimonie@163.com, bnwy1986@163.com, ${ }^{\mathrm{c}} 290505026 @ q q . c o m$ \\ *The corresponding author
}

Keywords: Phase-locked loop; Differential mode; Common mode; Maximum ratio combining; Symmetric diversity

\begin{abstract}
In the aerospace measuring and controlling area, especially in rocket telemetry receiver system, polarization diversity is used to fight against polarization signal fading, improve channel reliability and improve the quality of the received signal. Firstly, mathematical models of phase-locked loop, differential mode loop and common loop were demenstrated. Then, theoretical analysis was given in detail and the results indicated that interaction of differential mode loop and common mode loop the carrier capture and telemetry demodulation function can be finished, which can provide theoretical support for further analyzing, simulating and designing diversity symmetric phase-locked loop.
\end{abstract}

\section{Introduction}

The radio signal will fade during transmitting by kinds of influence factors such as water, earth and metal surface reflection which are called multipath fading [1-3]. When the radio frequency signal passes through the vehicle motor exhaust plume, the signal fading also happens that is named fame fading [4]. Moreover, numerous small reflectors and refraction bodies caused by thunder and storms lead to the random fading [5, 6]. The signal amplitude fading caused by these factors is generally large than 20 30 dB and it may drop to zero in severe cases. If it happens, the signal interrupts. So methods should be studied to solve this problem. The frequency modulation symmetric polarized diversity is one of them to solve the problem, the key of which is the phase-locked loop [7, 8]. In this manuscript theoretical analysis was given in detail and the results indicated that interaction of differential mode loop and common mode loop the carrier capture and telemetry demodulation function can be finished [9, 10], which can provide theoretical support for further analyzing, simulating and designing diversity symmetric phase-locked loop.

\section{Theoretical Analysis}

Polarization diversity is adding two or more branches in polarization fading signals, which are independent or little correlation from each other, to obtain a result free from fading than any tributary signal. The purpose of diversity is to ensure that the signal is not interrupted which improves the reliability of the channel and improves the quality of the received signal.

Frequency Modulation Diversity by the Symmetrical Phase-locked Loop. In order to achieve the maximum ratio sum, both the frequency and the phase should be the same at summing point, which is a coherent synthesizer. The PCM-FM telemetry system widely used in the symmetric phase-locked loop diversity is shown in Fig. 1. 


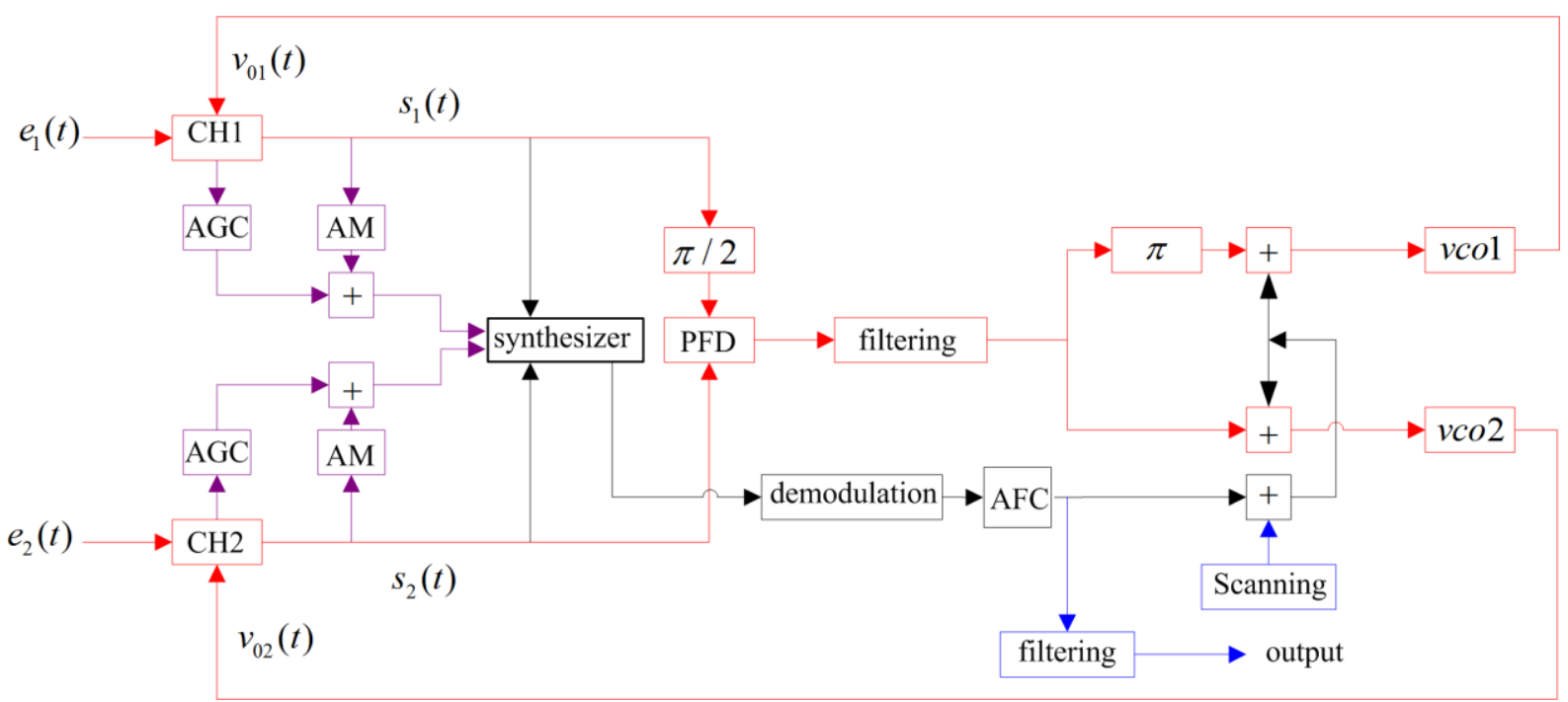

Figure 1. Schematic of frequency modulation symmetric phase-locked loop diversity

Due to the movement of the target and channel instability, there may be a slight frequency and phase differences between two channel's signals. Moreover, two VCO frequencies are also different in the process of work. Diversity must can separately adjusted two phase-locked loop's VCO frequency to achieve the two-way signal synthesizer coherent addition, so that the combined signal and the center frequency can be stable in the center of the loop bandwidth. FM diversity symmetrical phase-locked loop includes two main loops, which are called differential-mode loop actually a phase locked loop and the common mode loop actually an automatic frequency control circuit (AFC) loop.

In order to analyze the theoretical basis and functions of differential mode and common mode loops, the mathematical models are establishment as following.

Differential Mode Loop Mathematical Model. The principle of differential mode loop can be treated as a role for frequency driving. Firstly, the input signal can be written as following.

$\left\{\begin{array}{c}e_{1}=V_{i 1} \cos \left(\omega_{i 1} t+m_{f} \sin \Omega t+\varphi_{i 1}\right)=V_{i 1} \cos \left[\omega_{c} t+\varphi_{i 1}(t)\right] \\ e_{2}=V_{i 2} \cos \left(\omega_{i 2} t+m_{f} \sin \Omega t+\varphi_{i 2}\right)=V_{i 2} \cos \left[\omega_{c} t+\varphi_{i 2}(t)\right]\end{array}\right.$

Where $\left\{\begin{array}{l}\varphi_{i 1}(t)=\omega_{D o p 1} t+m_{f} \sin \Omega t+\varphi_{i 1} \quad \omega_{c} \text { is the carrier frequency, } \omega_{D o p 1} \text { and } \omega_{D o p 2} \text { are } \\ \varphi_{i 2}(t)=\omega_{D o p 2} t+m_{f} \sin \Omega t+\varphi_{i 2}\end{array}\right.$ branches Doppler frequency shift of the signals, $\varphi_{i 1}$ and $\varphi_{i 2}$ are channel phase shift and $m_{f} \sin \Omega t$ is signal modulation informations of the carried signals.

The output signals of the VCOs can be given as following.

$$
\left\{\begin{array}{l}
v_{01}(t)=V_{01} \cos \left\{\left[\omega_{c} t+\Delta \omega_{01}(t)\right] t+\varphi_{01}\right\} \\
v_{02}(t)=V_{02} \cos \left\{\left[\omega_{c} t+\Delta \omega_{02}(t)\right] t+\varphi_{02}\right\}
\end{array}\right.
$$

The phase of the phase detectors can be expressed as follows.

$$
\left\{\begin{array}{l}
\varphi_{e 1}(t)=\left(\omega_{D o p 1}-\Delta \omega_{01}\right) t+m_{f} \sin \Omega t+\varphi_{i 1}-\varphi_{01} \\
\varphi_{e 2}(t)=\left(\omega_{D o p 2}-\Delta \omega_{02}\right) t+m_{f} \sin \Omega t+\varphi_{i 2}-\varphi_{02}
\end{array}\right.
$$

Here phase detector is sinusoidal phase detector, so that there is half a pi phase shift. So we can get the outputs.

It should be noted that the input is the opposite polarization characteristics of the two signals, so the actual output error can be expressed as following.

$$
\varphi_{e}(t)=\left(\Delta \omega_{01}-\Delta \omega_{02}\right) t+\left(\omega_{D o p 1}-\omega_{D o p 2}\right) t+\left[\left(\varphi_{01}-\varphi_{02}\right)-\left(\varphi_{i 1}-\varphi_{i 2}\right)\right]
$$


Generally, $\varphi_{e}(t)$ may be larger or smaller than zero or eqaul to zero.

Firstly, when $\varphi_{e}(t)$ is smaller than zero, the control voltage after the inverter of VCO1is positive, so $\Delta \omega_{01}$ increases. At the moment the control voltage after the inverter of $\mathrm{VCO} 2$ is negative, so $\Delta \omega_{02}$ decreases. The result is that $\Delta \omega_{01}-\Delta \omega_{02}$ increases, which will make $\varphi_{e}(t)$ increasing towards the direction $\varphi_{e}(t)=0$.

Secondly, when $\varphi_{e}(t)$ is larger than zero, it will make $\varphi_{e}(t)$ deincreasing towards the direction $\varphi_{e}(t)=0$. Therefore it can be concluded that the differential mode loop is a negative feedback circuit, the results of which is to make a trend of $\varphi_{e}(t)=0$.

Common Mode Loop Mathematical Model. Common mode loop is comopsed of three parts, including a synthesizer, two VCOs and a frequency discriminator. The input signals of the synthesis can be expressed as following.

$$
\left\{\begin{array}{l}
s_{1}(t)=V_{s 1} \cos \varphi_{e 1}(t) \\
s_{2}(t)=V_{s 2} \cos \varphi_{e 2}(t)
\end{array}\right.
$$

Because of the frequency driving function of the differential mode loop, the synthesizer's output phase can be written as following.

$$
\left.\varphi_{\Sigma}(t)=\int\left[\omega_{D o p 2}-\Delta \omega_{02}\right)+\Omega m_{f} \cos \Omega t\right] d t+\varphi_{e}
$$

Signal after frequency discriminator can be written as following.

$$
v(t)=\frac{d \varphi_{\Sigma}(t)}{d t}=\Delta \omega+\Omega m_{f} \cos \Omega t
$$

After the AFC circuit filter, the control voltage output can be expressed as following.

$$
v_{c}(t)=\Delta \omega
$$

When $\Delta \omega$ is smaller than zero, the output frequencies of the VCOs decreased at the same control voltage, which will result in $\Delta \omega$ becoming larger. When $\Delta \omega$ is larger than zero, the output frequencies of the VCOs increased at the same control voltage, which will result in $\Delta \omega$ becoming smaller. And ultimately making $\Delta \omega$ is at near zero. Therefore it can be concluded that common mode loop is an automatic frequency control (AFC) circuit, the function of which is to make $\Delta \omega$ becoming zero.

\section{Conclusions}

Common mode and differential mode loop ring having frequency driving action. Differential mode loop in the opposite direction to drive two VCO, two VCO phase retardation value tracking values of two input signals, such signals in two branches before the synthesizer same frequency and phase coherent sum. Common mode loop in the same direction as the driving frequency of the VCO to ensure that two carrier frequency signal falls two branches at the center frequency of the loop filter, the two work together to ensure that the carrier capture and telemetry demodulation, improve channel reliability and improve the quality of the received signal. Common mode loop and the mathematical model of differential ring accurately describe the frequency of the drive ring and differential effects of common mode loop for further analysis, simulation and design diversity symmetric phase locked loop provides a theoretical basis.

\section{References}

[1] W. Rummler, R. Coutts and M. Liniger. "Multipath fading channel models for microwave digital radio" [J]. IEEE Communications Magazine, 1986, 24(11):30-42. 
[2] P.A. Molchanov, J.T. Astola K.O. Egiazarian and et al. "A novel bispectrum-based frequency encoding technique developed for noisy and multipath fading radio links" [J]. Telecommunications \& Radio Engineering, 2014, 73(3):221-239.

[3] F. Ishizu, K.Ueda, K. Murakami and et al. "A design of an anti-multipath fading modem for mobile satellite and land mobile communication systems" [C]. 14th International Communication Satellite Systems Conference and Exhibit, 2014, 1811-1816.

[4] T. Qi and Y. Wang. "Capacity Analysis of a Land Mobile Satellite System Using Dual-Polarized Antennas for Diversity’ [C]. IEEE Vehicular Technology Conference, 2015, 1-5.

[5] G.B. Giannakis and S. Zhou. "Optimal Transmit-Diversity Procoders for Random Fading Channels" [C]. Global Telecommunications Conference, 2000, 3:1839-1843.

[6] J. Lu, X. Wang and L. Zhang. "Signal power random fading based interference-aware routing for wireless sensor networks" [J]. Wireless Networks, 2014, 20(7):1715-1727.

[7] S. Yang, K.M. Luk, H.W. Lai and et al. "A dual-polarized antenna with pattern diversity" [J]. IEEE Antennas \& Propagation Magazine, 2009, 50(6):71-79.

[8] Z. Kui, J.S. Han, J.M. Choi and et al. "Advanced Preamble Transmit Diversity of Polarized DVB-T2 MISO System Using Hybrid Differential Modulation" [J]. IEEE Transactions on Broadcasting, 2015, 61(4):1-5.

[9] Y. J. Wu, G. Fu and G. Cheng. Pulse Coding Soft FM Telemetry Signal Demodulation, Advanced Materials Research, 2014, 989-994:3952-3955.

[10]D. X. Zhu, G. Fu and Y. Feng. Study of PCM/FM Telemetry Sequence Soft Demodulation, Advanced Materials Research, 2014, 989-994:3922-3925. 